\title{
Cirrus High-Definition Optical Coherence Tomography Compared with Stratus Optical Coherence Tomography in Glaucoma Diagnosis
}

\author{
Javier Moreno-Montañés, Natalia Olmo, Aurora Alvarez, Noelia García, and \\ Javier Zarranz-Ventura
}

\begin{abstract}
Purpose. To compare the retinal nerve fiber layer (RNFL) evaluation using Cirrus optical coherence tomography (OCT) and Stratus OCT in glaucoma diagnosis.
\end{abstract}

Methods. One hundred thirty normal and 86 patients with glaucoma were included in this prospective study. The signal strengths of the OCTs were evaluated. The sensitivities and specificities of global RNFL average thickness were compared in the four quadrants and in each clock hour sector. Receiver operating characteristic (ROC) curves, areas under the ROC (AUC), and the likelihood ratio (LR) were plotted for RNFL thickness. Agreement between the OCTs was calculated by using the Bland-Altman method and kappa $(\kappa)$ coefficient.

REsults. Twenty-three percent of all cases examined with Stratus OCT and $1.9 \%$ examined with Cirrus OCT had a signal strength below $6(P=0.01)$. In cases with signal strengths $\geq 6$, the mean signal strength was higher with Cirrus OCT than with Stratus OCT $(P=0.01)$. The RNFL measurements by Cirrus were thicker than those of Stratus OCT $(P<0.05)$. The AUCs were 0.829 for Stratus and 0.837 for Cirrus OCT $(P=0.706)$ for global RNFL average. LRs were similar in both OCTs in global RNFL classification but varied in quadrants. The widths of the limits of agreement varied between 42.16 and $97.79 \mu \mathrm{m}$. There was almost perfect agreement $(\kappa=0.82)$ in the average RNFL classification.

Conclusions. Cirrus OCT has better scan quality than Stratus OCT, especially in glaucomatous eyes. In cases with goodquality scans, the sensitivity and specificity, and AUCs were similar. The best agreement was in the global average RNFL classification. The widths of limits of agreements exceed the limits of resolution of the OCTs. (Invest Ophthalmol Vis Sci. 2010;51:335-343) DOI:10.1167/iovs.08-2988

$\mathrm{O}$ ptical coherence tomography (OCT) is an optical imaging technique that provides high resolution, cross-sectional, in vivo measurements of the retinal nerve fiber layer (RNFL) in a fashion similar to B-scan ultrasonography. Since OCT was introduced in $1991,{ }^{1}$ several ocular applications have been developed. Different OCT systems have been introduced re-

From the Department of Ophthalmology, Clínica Universidad de Navarra, Pamplona, Spain.

Supported in part by RETIC (Cooperative Research Thematic Networks) Grant RD 07/0062 (Oftalmología), Instituto de Salud Carlos III, Madrid, Spain.

Submitted for publication October 9, 2008; revised March 31, June 26, and July 24, 2009; accepted August 22, 2009.

Disclosure: J. Moreno-Montañés, None; N. Olmo, None; A. Alvarez, None; N. García, None; J. Zarranz-Ventura, None

Corresponding author: Javier Moreno-Montañés, Department of Ophthalmology, Clínica Universidad de Navarra, Apartado 4209, 31080 Pamplona, Spain; jmoreno@unav.es. cently for quantitative and qualitative analysis of the RNFL, and use glaucoma algorithms to measure the thickness along a 3.4-mm-diameter circle centered on the optic disc.

Stratus OCT 3000 (Carl Zeiss Meditec, Dublin, CA) is a third-generation model with approximately 7 - to $8-\mu \mathrm{m}$ resolution. ${ }^{2}$ The RNFL appears graphically as a double hump, and the system also shows the global average and averages for each quadrant and clock hour position. Each average is classified as normal, borderline, or abnormal based on comparison with a normative database, with normal and glaucomatous cases distributed by age. ${ }^{3}$

New high-definition (HD) OCTs recently have become available. Spectral-domain OCT provides faster scanning than previous OCTs, allowing three-dimensional imaging of retinal tissue. Carl Zeiss Meditec introduced a spectral-domain OCT system, Cirrus, that offers faster scanning and better axial resolution than its predecessor, Stratus. Cirrus is an HD OCT system with an axial resolution of $6 \mu \mathrm{m}$ and a scanning speed of up to 25,000 A-scans/s. ${ }^{4}$ The scanning area covers $6 \times 6$ $\mathrm{mm}$, and the scanning depth is $2 \mathrm{~mm} .{ }^{4}$ Because of spectraldomain technology, Cirrus OCT provides a twofold higher resolution than Stratus OCT generates during the same time frame. Like Stratus, Cirrus analyzes a 3.4-mm-diameter circle automatically centered on the optic disc inside a $6 \times 6$ map and compares the results with those in a normal database.

The goal of the present study was to compare the RNFL measurements and RNFL classification by using the database between the Cirrus and Stratus OCTs in normal and glaucomatous eyes. We also compared the quality of the RNFL measurement between the OCTs.

\section{Methods}

\section{Subjects}

Normal eyes and eyes with glaucoma were recruited prospectively in the Department of Ophthalmology, Clínica Universidad de Navarra, Pamplona, Spain. The study was approved by the institutional review board/ethics committee. According to this committee no written informed consent was needed in the glaucoma group, because this evaluation was performed on data that are normally obtained in clinical practice. All normal volunteers provided informed consent before entering the study, which adhered to the Declaration of Helsinki.

Complete ophthalmic examinations of all participants were performed that included slit lamp biomicroscopy, intraocular pressure (IOP) measurement, dilated stereoscopic fundus examination, gonioscopy, and standard automated perimetry using the 24- 2 Swedish Interactive Threshold Algorithm (Humphrey Field Analyzer; Carl Zeiss, Meditec). All participants were Caucasian and had a spherical equivalent within $5.0 \mathrm{D}$ or less and astigmatism of $3.00 \mathrm{D}$ or less, a best corrected visual acuity (VA) of $20 / 40$ or better ( $\leq 0.3 \log$ MAR), no corneal or retinal disease, no history of amblyopia, no contraindication 
to dilation or intolerance of topical anesthetic or mydriatic agents, and no substantial media opacity that obscured the fundus.

The glaucoma group included cases with a clinical diagnosis of open-angle glaucoma identified by gonioscopy and visual field defects. The IOP exceeded $21 \mathrm{~mm} \mathrm{Hg}$ on at least three different days. The visual field was classified as glaucomatous according to a glaucoma staging system (GSS) that was a modified version of the Hodapp-AndersonParrish classification. ${ }^{5}$ This classification allows for assignments of glaucoma stage on the basis of visual field damage. The staging system assigns patients with glaucoma to different stages of disease progression on the basis of a combination of mean defect (MD) score and one of the following: pattern deviation probability plot score (indicating deviation from a normalized visual field pattern); decibel plot (stages 2-4) or; for stage 1, either corrected pattern SD/pattern SD (CPSD/ PSD) or glaucoma hemifield test results. The stages are 0 (ocular hypertension), 1 (early glaucoma), 2 (moderate glaucoma), 3 (advanced glaucoma), 4 (severe glaucoma), 5 (blindness). This visual field damage was reproducible in at least three reliable and consecutive visual fields performed on different days. In cases of bilateral glaucoma, only a randomized eye was included. The normal group included patients with an IOP of $21 \mathrm{~mm} \mathrm{Hg}$ or lower, normal visual fields, and no familial glaucoma history. This normal group included patients consecutively recruited from hospital staff, nurses, relatives of patients, and patients referred for a routine VA examination without ocular diseases.

A total of 216 eyes (106 right and 110 left eyes) of 216 patients (109 men, 107 women) were enrolled. The mean age \pm SD was $59.57 \pm$ 18.43 years. One hundred thirty eyes were normal and 86 were glaucomatous. Mean age in the normal group was $58.22 \pm 10.85$ years and, in the glaucomatous group, $60.12 \pm 12.45$ years $(P=0.285)$.

\section{Observation Procedures}

After pupillary dilation with $1 \%$ tropicamide, OCT evaluation using Stratus and Cirrus was performed on the same day. Before the study, both OCTs were calibrated by the manufacturer. The OCT examinations in both instruments were performed by an experienced operator who was different from the examiner who performed the visual field testing and was masked to the other findings. The first 30 examinations with the Cirrus OCT were not included in the analysis and were considered part of the learning curve.

Stratus OCT was performed first because it requires more cooperation from the patient than does Cirrus OCT, as observed during the learning curve with Cirrus OCT.

Stratus OCT Examination. The eyes were measured with the peripapillary fast RNFL program. When the fast RNFL program is used, the RNFL thickness is determined at 256 points around a 3.4-mm diameter around the center of the optic disc. RNFL data were analyzed automatically with the system software (version 4.0.1 software; Carl Zeiss Meditec). Three scans were taken, and only the best quality scan was included and the signal strength of the scan recorded.
Cirrus OCT Examination. Three OCT volume scans $(200 \times$ 200 axial scans) centered on the optic disc were acquired for each eye, and the scan with the best signal strength was selected. RNFL data were evaluated automatically by Cirrus OCT system software (version 3.0 software; Carl Zeiss Meditec) and the signal strength was analyzed.

The RFNL parameters evaluated in both OCTs were the global RNFL average (in micrometers) and RNFL thickness in four quadrants and in 12 clock hour positions. RNFL defects in clock hours were assessed clockwise in the right eyes and counterclockwise in the left eyes. RNFL measurements in quadrants were evaluated in the four $90^{\circ}$ sectors: superior, inferior, nasal, and temporal. The software in both OCTs automatically classified all RFNL values into three groups: within normal limits (green), borderline (yellow), and outside normal limits (red). The numbers of cases with signal strength below 6 were counted for both OCTs. Only cases with signal strength of 6 or higher were included in the sensitivity and specificity analysis and the comparison between RNFL measurements obtained by both OCTs. A qualitative OCT evaluation was performed considering borderline cases as positive (least specific criteria) and as negative (most specific criteria). Agreement between the qualitative RNFL classifications using both devices also was assessed considering three possible results: complete agreement (same classification group), partial agreement (classified by one analysis as borderline and by the other as either within normal limits or outside normal limits), and no agreement.

\section{Statistical Analysis}

Data were evaluated with commercial software (SPSS, ver. 15.0.1; SPSS Inc, Chicago, IL, and MedCalc, ver. 9.2; MedCalc Software, Mariakerke, Belgium). The Shapiro-Wilk test was used to assess the normality of the quantitative variables. Normally distributed variables were summarized by using the mean and $\mathrm{SD}$, and nonnormally distributed variables were summarized by using the median and interquartile ranges (IR; percentiles 25 and 75). The association between Cirrus and Stratus OCT was tested by determining Spearman's correlation coefficient $\left(r_{\mathrm{s}}\right)$. The quantitative variables between two independent groups (the sexes and normal and glaucomatous groups) were compared by using the MannWhitney U test nonnormally distributed variables (all but age), and two-sample Student's $t$-test was used for normally distributed variables (age only). To compare qualitative variables between independent groups, $\chi^{2}$ tests were used. RNFL sensitivities and specificities were calculated for both OCTs compared with visual field classification. Receiver operating characteristic (ROC) curves were used to determine the discriminatory capabilities between healthy and glaucomatous eyes. The areas under the ROC curves (AUC) were compared by using the Hanley-McNeil method for paired data. ${ }^{6} \mathrm{~A}$ difference between AUCs of 0.1 was considered to be clinically relevant. Using statistical software (Medcalc), a sample size of 57 eyes per group (control/glaucomatous) was estimated to detect a difference in AUC over 0.1 with a power of $95 \%$ at a significance level of $5 \%$; for a power of $90 \%$, a sample of 48 was needed. The sensitivities for $85 \%$ and $95 \%$

TABLE 1. Quality of Scans in Normal and Glaucomatous Eyes

\begin{tabular}{|c|c|c|c|}
\hline & Normal Cases & Glaucoma Cases & All Cases \\
\hline Cases, $n$ & 130 & 86 & 216 \\
\hline Signal strength with Stratus OCT in all eyes* & $6.34 \pm 1.01$ & $6.10 \pm 1.02$ & $6.26 \pm 1.1$ \\
\hline Signal strength with Cirrus OCT in all eyes* & $7.95 \pm 1.09 \dagger$ & $7.51 \pm 0.91 \dagger$ & $7.77 \pm 1.04 \dagger$ \\
\hline Cases with quality $<6$ with Stratus OCT, $n$ (\%) & $24(15.5) \ddagger$ & $25(29.1)$ & $49(22.7)$ \\
\hline Cases with quality $<6$ with Cirrus OCT, $n$ (\%) & $3(2.3) \dagger$ & $1(1.2) \dagger$ & $4(1.9) \dagger$ \\
\hline Cases with quality < 6 with both OCTs, $n$ (\%) & $2(1.5)$ & $1(1.2)$ & $3(1.4)$ \\
\hline Signal strength* with Stratus OCT in eyes with quality $\geq 6$ & $6.79 \pm 0.87$ & $6.58 \pm 0.80$ & $6.66 \pm 0.85$ \\
\hline Signal strength* with Cirrus OCT in eyes with quality $\geq 6$ & $8.20 \pm 0.90 \dagger \ddagger$ & $7.72 \pm 0.79 \dagger$ & $8.02 \pm 0.89 \dagger$ \\
\hline
\end{tabular}

Better quality is indicated by higher signal strength.

$*$ Mean \pm SD.

$\dagger P<0.05$ compared with Stratus OCT.

$\ddagger P<0.05$ compared with glaucoma cases. 
TABLE 2. Comparison of Cirrus and Stratus OCT Parameters for the Glaucoma Stages of the GSS 5

\begin{tabular}{|c|c|c|c|c|c|}
\hline & Stage 1 & Stage 2 & Stage 3 & Stage 4 & $\boldsymbol{P}$ \\
\hline Eyes, $n$ & 35 & 21 & 14 & 16 & \\
\hline Age, $y^{*}$ & $70.54 \pm 10.25$ & $66.80 \pm 14.68$ & $65.14 \pm 11.46$ & $72.5 \pm 7.97$ & 0.28 \\
\hline $\mathrm{MD}^{*}$ & $-3.0 \pm 1.21$ & $-7.81 \pm 2.01$ & $-14.7 \pm 1.32$ & $-26.14 \pm 2.88$ & 0.001 \\
\hline Signal strength in Stratus OCT $^{*}$ & $6.4 \pm 1.16$ & $5.9 \pm 0.88$ & $6.29 \pm 0.99$ & $5.56 \pm 0.36$ & 0.031 \\
\hline Signal strength in Cirrus OCT ${ }^{*}$ & $7.51 \pm 1.01 \dagger$ & $7.33 \pm 0.91 \dagger$ & $7.71 \pm 0.82 \dagger$ & $7.56 \pm 0.81 \dagger$ & 0.496 \\
\hline \multicolumn{6}{|l|}{ Cases with signal strength $<6$ with } \\
\hline Stratus OCT, $n(\%)$ & $6(17.1)$ & $7(33.3)$ & $4(28.6)$ & $8(50)$ & 0.11 \\
\hline \multicolumn{6}{|l|}{ Cases with signal strength $<6$ with } \\
\hline Cirrus OCT, $n(\%)$ & $1(2.9)$ & 0 & 0 & 0 & 0.66 \\
\hline \multicolumn{6}{|l|}{ Global RNFL average using Stratus } \\
\hline OCT (all cases)* & $74.13 \pm 17.10$ & $55.49 \pm 16.24$ & $51.61 \pm 17.54$ & $43.60 \pm 6.80$ & 0.001 \\
\hline \multicolumn{6}{|l|}{ Global RNFL average using Cirrus } \\
\hline OCT (all cases)* & $79.34 \pm 17.69 \dagger$ & $64.57 \pm 12.87 \dagger$ & $66.64 \pm 14.24 \dagger$ & $61.18 \pm 6.02 \dagger$ & 0.001 \\
\hline
\end{tabular}

$*$ Mean \pm SD.

$+P<0.05$ compared with Stratus OCT.

fixed specificities were calculated. Diagnostic classification of both OCT (outside normal limits, borderline, or within normal limits) provided by each instrument after comparison with its respective normative database was also evaluated, and likelihood ratios (LRs) were reported. The agreement of the global RNFL values (quantitative data) was evaluated by using the Bland-Altman method. ${ }^{7}$ The agreement of the RNFL classification (qualitative data) between both OCTs was assessed using the weighted kappa $(\kappa)$ coefficient. ${ }^{8}$ The strength of the qualitative agreement was categorized according to the method of Landis and Koch ${ }^{9}$ : less than 0 , no agreement; 0 to 0.20 , poor; 0.21 to 0.40 , low; 0.41 to 0.60 , moderate; 0.61 to 0.80 , substantial; and 0.81 to 1.00 , almost perfect.

\section{Results}

\section{Image Quality with the OCT Instruments}

Table 1 shows the quality of the RNFL measurements using both OCTs in normal and glaucomatous eyes. The mean signal strength was higher in Cirrus OCT than in Stratus OCT in the normal and glaucomatous groups $(P<0.01)$. Twenty-three percent of cases $(n=49)$ had a signal strength of 5 or less with Stratus OCT and $1.9 \%(n=4)$ with Cirrus OCT $(P=0.01)$. Older age was related to worse signal strength with Cirrus OCT
$(P<0.01)$, but not with Stratus OCT $(P=0.052)$. In normal eyes signal strength was not related to global RNFL measurements obtained with Cirrus OCT $(P=0.655)$. In eyes with glaucoma, signal strength was not related to the global RNFL measurements obtained by Cirrus OCT $(P=0.465)$. Finally, in all cases, signal strength was not related to global RNFL measurements $(P=0.185)$.

In Table 2 the MDs of the visual field and the signal strength in the glaucoma stages are included. The signal strength decreased according to the increase in glaucoma stage with Stratus OCT $(P=0.031)$, but not with Cirrus OCT $(P=0.496)$. In all stages, the signal strength was higher with Cirrus OCT than with Stratus OCT $(P<0.05)$.

After eliminating cases with signal strength of less than 6 , we had 166 cases with both OCTs with good quality scans (105 normal eyes, 61 glaucomatous eyes). In this group, the mean signal strength was higher with Cirrus OCT than with Stratus OCT in normal and glaucomatous eyes and in all eyes $(P<0.01$; Table 1$)$. In all cases, $136(81.93 \%)$ eyes had a higher signal strength with Cirrus than Stratus OCT; in 20 $(12.04 \%)$ cases, the signal strength was the same with both OCTs, and in $10(6.02 \%)$ cases; the signal strength was higher with Stratus than Cirrus OCT. The signal strength

TABLE 3. RNFL Thickness Measurements by Stratus and Cirrus OCTs

\begin{tabular}{lcrll}
\hline & Stratus OCT & Cirrus OCT & $\boldsymbol{r}_{\mathbf{s}}$ & $\boldsymbol{P}$ \\
\hline Global average & $78.83 \pm 20.28$ & $84.90 \pm 18.95$ & 0.88 & 0.001 \\
Quadrant & & & \\
$\quad$ Superior & $98.68 \pm 29.82$ & $103.40 \pm 26.00$ & 0.83 & 0.001 \\
Inferior & $93.22 \pm 31.56$ & $105.40 \pm 29.59$ & 0.82 & 0.001 \\
Temporal & $57.11 \pm 15.12$ & $59.06 \pm 13.98$ & 0.75 & 0.001 \\
Nasal & $66.34 \pm 18.87$ & $68.28 \pm 12.79$ & 0.73 & 0.001 \\
Clock hour & & & & \\
1 & $91.22 \pm 32.55$ & $95.49 \pm 25.87$ & 0.82 & 0.001 \\
2 & $79.12 \pm 24.65$ & $82.17 \pm 18.65$ & 0.73 & 0.004 \\
3 & $56.58 \pm 18.09$ & $60.14 \pm 14.26$ & 0.61 & 0.001 \\
4 & $62.75 \pm 22.32$ & $63.25 \pm 15.63$ & 0.60 & 0.02 \\
5 & $83.31 \pm 30.71$ & $89.54 \pm 25.29$ & 0.77 & 0.001 \\
6 & $100.11 \pm 37.60$ & $115.56 \pm 38.14$ & 0.79 & 0.001 \\
7 & $96.41 \pm 37.96$ & $110.46 \pm 37.16$ & 0.78 & 0.001 \\
8 & $57.45 \pm 20.55$ & $60.88 \pm 18.60$ & 0.63 & 0.001 \\
9 & $46.27 \pm 12.16$ & $49.19 \pm 13.25$ & 0.57 & 0.07 \\
10 & $67.16 \pm 20.25$ & $67.73 \pm 18.56$ & 0.76 & 0.001 \\
11 & $101.55 \pm 31.746$ & $104.10 \pm 31.89$ & 0.83 & 0.001 \\
12 & $103.58 \pm 36.37$ & $110.36 \pm 33.27$ & 0.74 & 0.001 \\
\hline
\end{tabular}

Data are expressed as the mean $\pm \mathrm{SD} . r_{\mathrm{s}}$, Spearman's rho. 


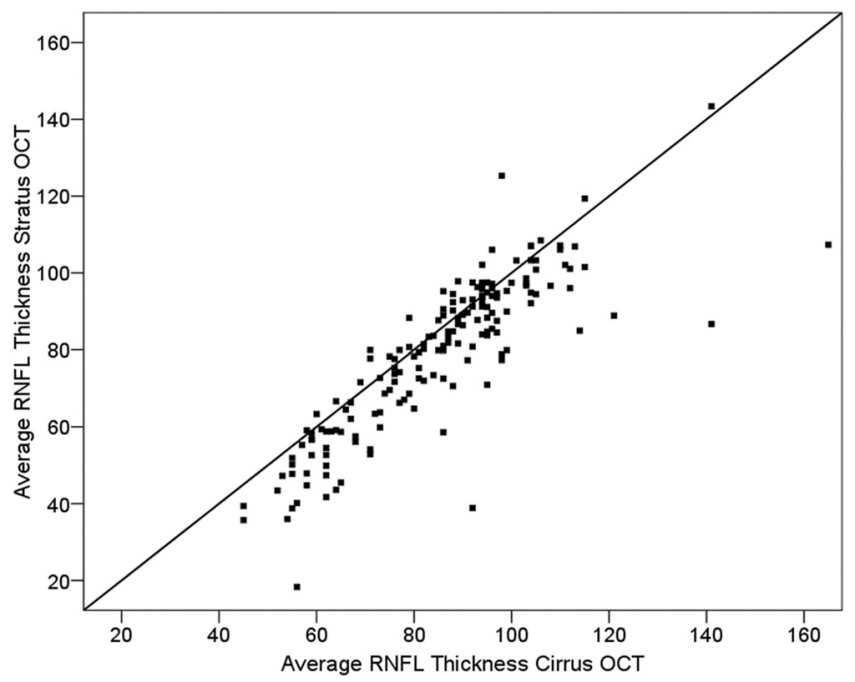

FigURE 1. Global average RNFL thickness measurement comparison between Stratus and Cirrus OCT.

difference between both OCTs was related to age $(P=$ $0.001)$.

\section{Comparison of RNFL Measurements}

Table 3 shows the mean \pm SD of RNFL thickness with both OCTs and the Spearman correlation coefficient. This correlation coefficient was significant in the global RNFL average and in all quadrants and clock hours evaluated, except at the 9 o'clock position $\left(r_{\mathrm{s}}=0.57 ; P=0.07\right)$. At the 1 o'clock and 11 o'clock positions, the $r_{\mathrm{s}}$ were higher than in other clock hour sectors. The global RNFL measurements average in Cirrus OCT was higher than with Stratus OCT in 127 (76.5\%) eyes and lower in 39 (23.5\%) eyes (Fig. 1). These differences between the OCTs were unrelated to differences in signal strength $(P=$ $0.29)$, age $(P=0.24)$, sex $(P=0.94)$, or spherical equivalent $(P=0.64)$.

In Table 2 the global RNFL average is compared in both OCTs at each stage of glaucoma. In both OCTs, the global RNFL average decreased as the glaucoma progressed to higher stages $(P<0.05)$. In all stages the global RNFL average was higher with Cirrus OCT than with Stratus OCT $(P<0.05)$.

The difference (Cirrus OCT minus Stratus OCT) was plotted against the average of the two measurements by using BlandAltman plots (Table 4). The scatterplots showed that the RNFL thickness measurements were thicker with Cirrus OCT than with Stratus OCT in all quadrant or hour positions. The data distribution showed that seven (4.21\%) eyes were outside the normal limits in global average RNFL (Fig. 2). In addition, inspection of the plots revealed a wide level of agreement in RNFL thickness measurements (width of limits of agreement [LoA] between $42.16 \mu \mathrm{m}$ in global average RNFL and $97.79 \mu \mathrm{m}$ at the 12 o'clock position) obtained by the two instruments.

\section{Sensitivity and Specificity in Glaucoma Detection}

Table 5 shows the best sensitivity-specificity balance, sensitivity for $85 \%$ and $95 \%$ fixed specificities, and AUCs of RNFL thickness for discriminating between healthy and glaucomatous eyes with Stratus OCT. Table 6 shows the AUCs, sensitivity, specificity, and AUCs of RNFL thickness with Cirrus OCT. In relation to Cirrus OCT, the best sensitivity for a fixed specificity was the global RNFL average. In the quadrants, the best AUC was in the superior quadrant. Regarding the clock hour positions, all AUCs were significant except the 9 o'clock position. The sensibility and specificity of glaucoma detection in both OCTs did not change if there was at least one abnormal quadrant. Thus, in eyes with at least one abnormal quadrant the sensibility and specificity was $68.9 \%$ and $89.5 \%$ with the Stratus OCT and $70.5 \%$ and $84.8 \%$ with the Cirrus OCT.

The AUCs were compared between both OCT in the initial stages of glaucoma (stages 1 and 2 of GSS classification); the AUC for the global RNFL average with Stratus OCT was 0.796 and with Cirrus OCT, $0.776(P=0.907)$. In cases of more advanced glaucoma (stages 3 and 4 of GSS), ${ }^{5}$ the AUCs for the global RNFL average were 0.956 and 0.934 with Stratus OCT and Cirrus OCT, respectively $(P=0.297)$.

The LRs with their 95\% confidence intervals (CIs) for RNFL classification after comparison with the instrument's normative database are presented in Tables 7 and 8 . The results for the global RNFL average were similar with both OCT systems. Thus, the LR $(95 \% \mathrm{CI})$ for outside normal limits was 7.08 (3.67-13.64) for Stratus-OCT and $7.87(3.70-16.73)$ for Cirrus

TABLE 4. Bland-Altman Analysis of RNFL Thickness Measurements by Stratus and Cirrus OCTs

\begin{tabular}{|c|c|c|c|c|}
\hline & \multirow{2}{*}{$\begin{array}{c}\text { Mean Difference } \pm \text { SD } \\
\text { (Cirrus OCT Minus Stratus OCT) }\end{array}$} & \multicolumn{3}{|c|}{ 95\% LoA } \\
\hline & & Lower LoA & Upper LoA & Width of LoA \\
\hline Average & $6.06 \pm 10.75$ & -15.01 & 27.15 & 42.16 \\
\hline \multicolumn{5}{|l|}{ Quadrant } \\
\hline Superior & $4.72 \pm 15.69$ & -26.03 & 35.47 & 61.5 \\
\hline Inferior & $12.17 \pm 17.17$ & -21.48 & 45.83 & 67.31 \\
\hline Temporal & $1.94 \pm 12.16$ & -21.89 & 25.78 & 47.67 \\
\hline Nasal & $1.94 \pm 12.56$ & -22.69 & 26.58 & 49.27 \\
\hline \multicolumn{5}{|l|}{ Clock hour } \\
\hline 1 & $4.27 \pm 19.71$ & -34.37 & 42.92 & 77.29 \\
\hline 2 & $3.04 \pm 17.53$ & -31.31 & 37.41 & 68.72 \\
\hline 3 & $3.56 \pm 15.16$ & -26.16 & 33.29 & 59.45 \\
\hline 4 & $0.49 \pm 18.44$ & -35.66 & 36.64 & 72.30 \\
\hline 5 & $6.22 \pm 20.24$ & -33.45 & 45.91 & 79.36 \\
\hline 6 & $15.44 \pm 24.74$ & -33.04 & 63.93 & 96.97 \\
\hline 7 & $14.05 \pm 24.68$ & -34.31 & 62.42 & 96.73 \\
\hline 8 & $3.43 \pm 18.65$ & -33.12 & 39.99 & 73.11 \\
\hline 9 & $2.92 \pm 14.44$ & -25.39 & 31.23 & 56.62 \\
\hline 10 & $0.56 \pm 14.42$ & -27.71 & 28.84 & 56.55 \\
\hline 11 & $2.54 \pm 18.10$ & -32.92 & 38.02 & 70.94 \\
\hline 12 & $6.77 \pm 24.95$ & -55.67 & 42.12 & 97.79 \\
\hline
\end{tabular}




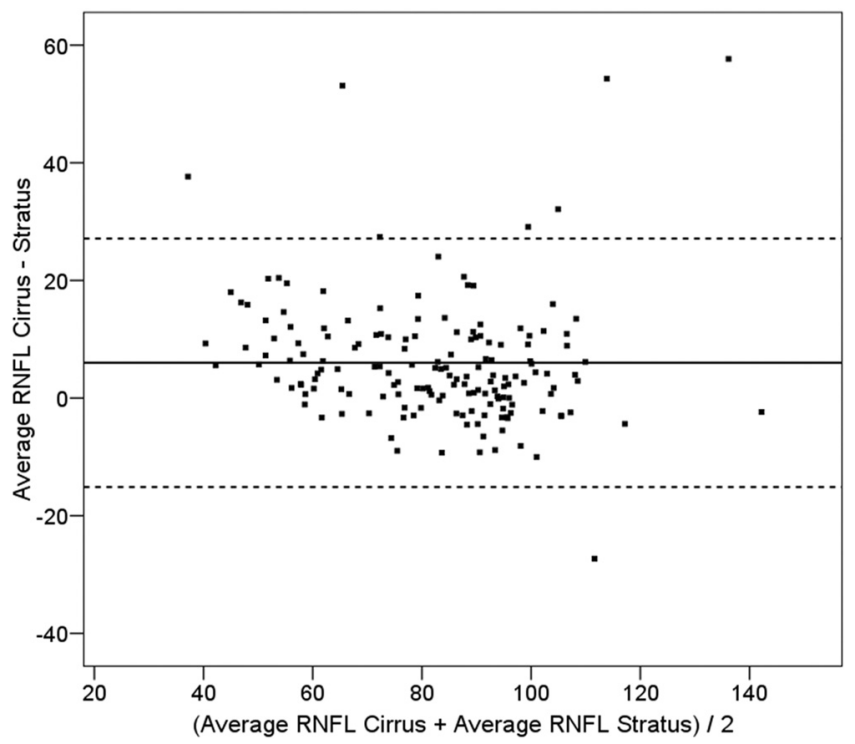

FiguRE 2. Bland-Altman plot comparing the global RNFL thickness measurements obtained with Cirrus and Stratus OCT. Mean difference \pm SD: RNFL measurement Cirrus minus Stratus measurement, $6.06 \pm 10.75 \mu \mathrm{m}$; limits of agreement, -15.01 to $27.15 \mu \mathrm{m}$.

OCT. Within-normal-limits results had an LR (95\% CI) of 0.36 (0.25-0.53) for Stratus OCT and 0.37 (0.25-0.53) for Cirrus OCT for the global RNFL average. For borderline results the LRs were higher with Cirrus OCT for global average and in all quadrants.

Table 9 shows the differences between the AUCs from the OCTs in RNFL thickness. In the global average RNFL thickness, the difference was not significant ( $P=0.706$; Fig. 3). A comparison of the quadrants between the OCTs showed differences in the temporal area $(P=0.003)$. Regarding clock hour sectors, the differences were also significant at four clock hours.

The RNFL classification agreement was evaluated by using the $\kappa$ coefficient (Table 10). The agreement was almost perfect in the global RNFL average classification $(\kappa=0.82)$. The best agreement in the quadrants was in the inferior and superior quadrants (substantial agreement). Regarding clock hour position, the agreement varied from 7 (substantial agreement) to 3 (slight agreement) o'clock.

\section{Discussion}

OCT has much potential for diagnosing glaucoma and glaucomatous progression. ${ }^{10}$ The level of discrimination (in micrometers) potentially makes OCT an objective tool for diagnosing axonal loss. This ability is important because RNFL damage precedes measurable visual field loss. ${ }^{11-13}$ The third-generation Stratus OCT quantifies RNFL thickness at a resolution of 8 to 10 $\mu \mathrm{m} .{ }^{14}$ Stratus OCT has a published sensitivity and specificity according to glaucoma development. Thus, DeLeón-Ortega et al. ${ }^{15}$ found a sensitivity and specificity of $77.22 \%$ and $80 \%$, Hood et al. ${ }^{16}$ reported $78 \%$ and $98 \%$, and Budenz et al. ${ }^{17}$ reported $89 \%$ and $92 \%$ in glaucoma with manifest visual field defects. In a previous study, we reported sensitivity/ and specificity of $80 \%$ and $74 \% .{ }^{18}$ New-generation HD OCT was designed to improve glaucoma detection. Cirrus OCT is an HD OCT system with an axial resolution of $6 \mu \mathrm{m}$ and a reproducibility of $1.6 \mu \mathrm{m} .{ }^{4}$ Although recent studies have reported the usefulness of Cirrus OCT in macular diseases, ${ }^{19-21}$ to our knowledge, no previous studies have compared the diagnostic accuracy of Cirrus OCT and Stratus OCT for glaucoma.

In the present study, the mean signal strength with Stratus OCT was $6.1 \pm 1.02$ in glaucomatous eyes, and $29.1 \%$ of glaucomatous eyes had unreliable RNFL measurements, because the signal strength was less than 6 . These results are similar to those in other published articles. ${ }^{22-24}$ The OCT software assigns each scan a quality indicator score known as signal strength from 1 to 10 . This score is based on a combination of signal-to-noise ratio and the uniformity of the signal strength within a scan, but for commercial reasons the manufacturer does not provide further details. ${ }^{23,25}$ In most studies, the accepted minimum signal-to-noise ratio is $6 .^{10} \mathrm{Wu}$ et al. ${ }^{22}$ suggested that although the manufacturer recommends higher signal strengths for OCT scans, in clinical practice, it is not always possible to obtain a higher

TABLE 5. Discrimination between Healthy and Glaucomatous Eyes with Stratus OCT

\begin{tabular}{|c|c|c|c|c|c|}
\hline & \multirow[b]{2}{*}{ AUC $(95 \%$ CI) } & \multirow[b]{2}{*}{$\boldsymbol{P}$} & \multirow[b]{2}{*}{$\begin{array}{c}\text { Sensitivity/ } \\
\text { Specificity (\%) }\end{array}$} & \multicolumn{2}{|c|}{ Sensitivity by Specificity (\%) } \\
\hline & & & & $\begin{array}{c}85 \% \\
\text { Specificity }\end{array}$ & $\begin{array}{c}95 \% \\
\text { Specificity }\end{array}$ \\
\hline Global average & $0.829(0.763-0.883)$ & 0.0001 & $68.9 / 86.7$ & 68.81 & 57.38 \\
\hline \multicolumn{6}{|l|}{ Quadrant } \\
\hline Superior & $0.809(0.740-0.865)$ & 0.0001 & $73.8 / 77.1$ & 60.66 & 49.15 \\
\hline Inferior & $0.795(0.725-0.853)$ & 0.0001 & $62.3 / 93.3$ & 65.57 & 59.02 \\
\hline Temporal & $0.768(0.696-0.830)$ & 0.0001 & $62.3 / 89.5$ & 63.93 & 44.26 \\
\hline Nasal & $0.752(0.679-0.816)$ & 0.0001 & $54.1 / 87.6$ & 54.10 & 32.79 \\
\hline \multicolumn{6}{|l|}{ Clock hour } \\
\hline 1 & $0.762(0.690-0.824)$ & 0.0001 & $57.4 / 88.6$ & 59.02 & 40.98 \\
\hline 2 & $0.735(0.661-0.800)$ & 0.0001 & $47.5 / 92.4$ & 47.54 & 37.70 \\
\hline 3 & $0.697(0.621-0.766)$ & 0.0001 & $67.2 / 62.9$ & 37.70 & 19.67 \\
\hline 4 & $0.709(0.634-0.777)$ & 0.0001 & $45.9 / 87.6$ & 45.38 & 22.95 \\
\hline 5 & $0.735(0.661-0.800)$ & 0.0001 & $57.4 / 88.6$ & 57.38 & 37.70 \\
\hline 6 & $0.757(0.685-0.820)$ & 0.0001 & $62.3 / 84.8$ & 60.66 & 50.82 \\
\hline 7 & $0.818(0.751-0.873)$ & 0.0001 & $72.1 / 81.9$ & 65.57 & 55.74 \\
\hline 8 & $0.799(0.730-0.857)$ & 0.0001 & $72.1 / 79$ & 59.02 & 34.43 \\
\hline 9 & $0.662(0.585-0.733)$ & 0.0001 & $37.7 / 90.5$ & 39.34 & 33.79 \\
\hline 10 & $0.757(0.684-0.820)$ & 0.0001 & $60.7 / 89.5$ & 62.30 & 44.26 \\
\hline 11 & $0.792(0.722-0.851)$ & 0.0001 & $57.4 / 91.4$ & 63.93 & 42.62 \\
\hline 12 & $0.765(0.693-0.827)$ & 0.0001 & $77 / 66.7$ & 45.91 & 36.07 \\
\hline
\end{tabular}


TABLE 6. Discrimination between Healthy and Glaucomatous Eyes with Cirrus OCT

\begin{tabular}{|c|c|c|c|c|c|}
\hline & \multirow[b]{2}{*}{ AUC (95\% CI) } & \multirow[b]{2}{*}{$\boldsymbol{P}$} & \multirow[b]{2}{*}{$\begin{array}{c}\text { Sensitivity/ } \\
\text { Specificity (\%) }\end{array}$} & \multicolumn{2}{|c|}{ Sensitivity by Specificity (\%) } \\
\hline & & & & $\begin{array}{c}85 \% \\
\text { Specificity }\end{array}$ & $\begin{array}{c}95 \% \\
\text { Specificity }\end{array}$ \\
\hline Global average & $0.837(0.772-0.889)$ & 0.0001 & $80.3 / 79$ & 73.77 & 52.46 \\
\hline \multicolumn{6}{|l|}{ Quadrant } \\
\hline Superior & $0.838(0.774-0.891)$ & 0.0001 & $68.9 / 91.4$ & 70.49 & 50.82 \\
\hline Inferior & $0.827(0.761-0.881)$ & 0.0001 & $60.7 / 95.2$ & 68.85 & 60.66 \\
\hline Temporal & $0.679(0.602-0.749)$ & 0.0001 & $60.7 / 75.2$ & 42.62 & 22.95 \\
\hline Nasal & $0.742(0.668-0.807)$ & 0.0001 & $55.7 / 89.5$ & 57.38 & 18.03 \\
\hline \multicolumn{6}{|l|}{ Clock hour } \\
\hline 1 & $0.818(0.750-0.873)$ & 0.0001 & $70.5 / 85.7$ & 70.49 & 40.98 \\
\hline 2 & $0.758(0.685-0.821)$ & 0.0001 & $68.9 / 74.3$ & 50.82 & 22.90 \\
\hline 3 & $0.603(0.524-0.678)$ & 0.0213 & $62.3 / 57.1$ & 21.31 & 9.84 \\
\hline 4 & $0.644(0.566-0.716)$ & 0.0009 & $47.5 / 78.1$ & 31.15 & 10.48 \\
\hline 5 & $0.762(0.690-0.824)$ & 0.0001 & $72.1 / 70.5$ & 49.18 & 29.51 \\
\hline 6 & $0.804(0.735-0.861)$ & 0.0001 & $59 / 96.2$ & 67.21 & 60.06 \\
\hline 7 & $0.785(0.715-0.845)$ & 0.0001 & $65.6 / 89.5$ & 67.21 & 55.74 \\
\hline 8 & $0.633(0.555-0.707)$ & 0.0022 & $55.7 / 72.4$ & 32.79 & 13.11 \\
\hline 9 & $0.584(0.505-0.660)$ & 0.0616 & $34.4 / 84.9$ & 34.4 & 14.75 \\
\hline 10 & $0.745(0.672-0.810)$ & 0.0001 & 63.9/81.9 & 59.03 & 40.98 \\
\hline 11 & $0.795(0.725-0.853)$ & 0.0001 & $65.6 / 86.7$ & 65.57 & 54.10 \\
\hline 12 & $0.770(0.698-0.831)$ & 0.0001 & $68.9 / 79$ & 59.02 & 36.53 \\
\hline
\end{tabular}

signal in many patients. In the present study, with Cirrus OCT, the number of cases with a signal strength $<6$ was only $1.9 \%$. In most cases, the signal strength was higher with Cirrus than with Stratus OCT $(81.93 \%)$. These results suggested that Cirrus OCT takes a higher quality scan than Stratus OCT.

The differences in the present study in signal strength between the OCTs could result from various factors. Pupillary dilation can modify the signal strength. Smith et al. ${ }^{23}$ reported a mean signal strength of 5.24 with Stratus OCT in undilated pupils, whereas the mean dilated score was 6.09, and the acquisition of high-quality OCT images was impossible without pupillary dilation in approximately $25 \%$ of patients. ${ }^{23}$ The Stratus OCT manufacturer suggested that 3-mm pupillary dilation is needed. However, because the Cirrus OCT uses a line scanning ophthalmoscope (LSO) with a 750-nm superluminescent diode as a light source, the quality of the captured images may be better than those with Stratus OCT, because this LSO provides clear pupil visualization and fundus image focusing. Another cause of differences between the signal strength of the OCTs could be changes in the ocular media transparency. ${ }^{24}$ However, Cirrus OCT has an advanced optics aid when evaluating patients with cataracts. ${ }^{4}$ Finally, another possible cause could be that both OCTs have different methods of signal strength analysis, and a strength of 6 may not signify the same thing in both OCTs. Although determining the causes of low signal strength was not a study goal, we found that, with similar conditions of pupillary dilation or lens transparency, Cirrus OCT has better signal strength than Stratus OCT.

The mean RNFL thickness was superior in Cirrus OCT when compared with Stratus OCT. The reason for this difference in systems of different OCT generations could be that in each new OCT system the axial resolution is improved over that of

TABLE 7. Stratus OCT RNFL Classification

\begin{tabular}{lccc}
\hline Parameter & Within Normal Limits & Borderline & Outside Normal Limits \\
\hline Global average & $0.36(0.25-0.53)$ & $1.43(0.46-4.50)$ & $7.08(3.67-13.64)$ \\
Quadrant & & & \\
$\quad$ Superior & $0.52(0.40-0.69)$ & $1.97(0.75-5.16)$ & $13.77(4.32-43.84)$ \\
Inferior & $0.42(0.28-0.62)$ & $0.43(0.15-1.23)$ & $5.95(3.29-10.75)$ \\
Temporal & $0.49(0.37-0.66)$ & $2.75(1.33-5.68)$ & $30.98(4.24-226.39)$ \\
Nasal & $0.89(0.79-1.01)$ & $2.01(0.71-5.70)$ & $5.16(0.55-48.56)$ \\
Clock hour & & & \\
1 & $0.67(0.53-0.83)$ & $1.55(0.67-3.60)$ & $13.77(3.26-57.87)$ \\
2 & $0.83(0.72-0.95)$ & $3.10(1.09-8.82)$ & Infinity (NA) \\
3 & $0.89(0.80-1.00)$ & $2.41(0.79-7.26)$ & Infinity (NA) \\
4 & $0.88(0.76-1.02)$ & $1.72(0.76-3.90)$ & $5.16(0.55-48.56)$ \\
5 & $0.54(0.41-0.70)$ & $2.37(1.01-5.56)$ & $9.04(3.25-25.10)$ \\
6 & $0.46(0.32-0.65)$ & $1.26(0.62-2.57)$ & $6.89(3.20-14.81)$ \\
7 & $0.37(0.25-0.53)$ & $3.87(1.24-12.05)$ & $7.87(3.70-16.73)$ \\
8 & $0.55(0.42-0.71)$ & $2.95(1.23-7.09)$ & $8.18(2.92-22.93)$ \\
9 & $0.69(0.57-0.85)$ & $2.41(1.14-5.09)$ & $15.49(2.01-119.35)$ \\
10 & $0.57(0.45-0.73)$ & $2.71(1.11-6.61)$ & $15.49(3.72-64.50)$ \\
11 & $0.48(0.35-0.65)$ & $2.49(1.13-5.47)$ & $6.60(2.84-15.30)$ \\
12 & $0.69(0.58-0.84)$ & $4.73(1.58-14.22)$ & $8.61(1.95-38.00)$ \\
\hline
\end{tabular}

Data are expressed as LR (95\% CI). NA, not applicable. 
TABLE 8. Cirrus OCT RNFL Classification

\begin{tabular}{lccc}
\hline Parameter & Within Normal Limits & Borderline & Outside Normal Limits \\
\hline Global average & $0.37(0.25-0.53)$ & $3.87(1.24-12.05)$ & $7.87(3.70-16.73)$ \\
Quadrant & & & \\
$\quad$ Superior & $0.42(0.30-0.58)$ & $2.87(0.71-11.59)$ & $7.10(3.51-14.37)$ \\
Inferior & $0.39(0.28-0.57)$ & $0.86(0.22-3.32)$ & $7.96(3.97-15.97)$ \\
Temporal & $0.73(0.60-0.87)$ & $17.21(2.26-131.23)$ & $2.46(0.99-6.13)$ \\
Nasal & $0.92(0.83-1.01)$ & $2.58(0.76-8.79)$ & Infinity (NA) \\
Clock hour & & & \\
1 & $0.59(0.47-0.75)$ & $4.02(1.63-9.91)$ & $22.38(3.0-166.90)$ \\
2 & $0.94(0.87-1.03)$ & $1.72(0.36-8.26)$ & Infinity (NA) \\
3 & $0.97(0.91-1.03)$ & $2.58(0.44-15.02)$ & Infinity (NA) \\
4 & $0.92(0.83-1.03)$ & $2.07(0.66-6.48)$ & $3.44(0.32-37.18)$ \\
5 & $0.74(0.62-0.88)$ & $4.30(1.41-13.13)$ & $5.16(1.45-18.35)$ \\
6 & $0.45(0.33-0.60)$ & $12.05(1.52-95.62)$ & $12.05(4.44-32.72)$ \\
7 & $0.50(0.38-0.68)$ & $0.52(0.15-1.80)$ & $17.79(5.67-55.74)$ \\
8 & $0.84(0.71-0.99)$ & $1.72(0.76-3.90)$ & $3.44(0.89-13.27)$ \\
9 & $0.87(0.76-0.99)$ & $3.87(1.24-12.05)$ & $1.29(0.30-5.58)$ \\
10 & $0.65(0.52-0.81)$ & $2.46(0.99-6.13)$ & $8.61(2.59-28.54)$ \\
11 & $0.42(0.3-0.59)$ & $1.72(0.79-3.73)$ & $12.05(4.44-32.72)$ \\
12 & $0.83(0.72-0.95)$ & $2.41(0.79-7.26)$ & Infinity (NA) \\
\hline
\end{tabular}

Data are expressed as LR (95\% CI). NA, not applicable.

the previous generation. The difference can also result from intermachine variability. Sehi et al. ${ }^{26}$ compared RNFL thickness readings between two Stratus OCTs and found substantial differences between the instruments in average, superior, and inferior RNFL thickness and central foveal thickness. The intermachine variability was greater than interoperator variability for all Stratus OCT measurements. Another reason for the differences among the quadrants or the clock hour positions may be that there is no assurance that the scans can be obtained at exactly the same locations around the optic nerve. Finally, Sehi et al. suggested that unexplained sources of error (quality of superluminescent diode beams, artifacts) can contribute to measurement variability.

We assessed the diagnostic capacity of OCTs by using sensitivities and specificities and AUC comparisons. The sensitivity and specificity of the global average RNFL were similar in both instruments, and the AUC comparison between them was not significant $(P=0.706)$. Leal et al. ${ }^{27}$

TABLE 9. Difference in AUC between Cirrus and Stratus OCTs in RNFL Thicknesses

\begin{tabular}{llcc}
\hline & $\begin{array}{c}\text { Difference } \\
\text { between AUCs }\end{array}$ & $95 \%$ CI & $\boldsymbol{P}$ \\
\hline $\begin{array}{l}\text { Global average } \\
\text { Quadrant }\end{array}$ & 0.00804 & -0.0338 to 0.0499 & 0.706 \\
$\quad \begin{array}{l}\text { Superior } \\
\text { Inferior }\end{array}$ & 0.0299 & -0.0167 to 0.0765 & 0.208 \\
Temporal & 0.0324 & -0.0178 to 0.0826 & 0.206 \\
Nasal & 0.0888 & 0.0293 to 0.148 & 0.003 \\
Clock hour & 0.0102 & -0.0584 to 0.0788 & 0.770 \\
1 & & & \\
2 & 0.0558 & 0.00431 to 0.107 & 0.034 \\
3 & 0.0229 & -0.0417 to 0.0874 & 0.694 \\
4 & 0.0945 & 0.0105 to 0.178 & 0.027 \\
5 & 0.0657 & -0.0194 to 0.151 & 0.130 \\
6 & 0.027 & -0.0313 to 0.0853 & 0.364 \\
7 & 0.0466 & -0.00568 to 0.0989 & 0.081 \\
8 & 0.0328 & -0.0253 to 0.0908 & 0.001 \\
9 & 0.166 & 0.0932 to 0.239 & 0.001 \\
10 & 0.0777 & -0.00813 to 0.163 & 0.076 \\
11 & 0.0116 & -0.0477 to 0.0708 & 0.702 \\
12 & 0.00312 & -0.0461 to 0.0523 & 0.901 \\
& 0.00468 & -0.0566 to 0.0659 & 0.881 \\
\hline
\end{tabular}

compared AUCs from Stratus OCT and OCT-1 and found significantly greater values with Stratus OCT in the global average RNFL and in the temporal quadrant measurement in eyes with band atrophy of the optic nerve and temporal hemianopsia. Bourne at al. $^{28}$ compared AUCs from OCT 2000 and Stratus OCT and showed that the glaucoma discrimination of the global RNFL average was better with Stratus OCT than with OCT 2000. In the present study, the superior and inferior quadrants had the highest AUCs without differences between instruments $(P=$ 0.20). Other reports of OCT 2000 and Stratus OCT found that the superior and inferior quadrants had the best AUCs. ${ }^{17,28-30}$

In the present study, LRs for outside normal limits results with both OCT systems were generally associated with large changes from pre- to posttest probability of glaucoma. Borderline results were associated with small to moderate effects, whereas within-normal-limits results were associated with small effects. These results indicate that results for each of these tests would induce only a small change in the pretest probability of disease. The LR, which is independent of disease prevalence, provides an indication of how much the odds of disease change based on a positive or negative result. An LR over 10 or under 0.1 often indicates large changes in posttest odds of having the disease. ${ }^{31}$ Similar to Parikh et al., ${ }^{32}$ we did not identify a parameter with an LR that can be used to rule out glaucoma (LR $<0.1)$.

The study results indicated a low agreement from a clinical standpoint between the Stratus and Cirrus OCTs based on the variations in the RNFL thickness with LoA that were relatively wider and larger compared with the limits of resolution of both OCTs $(5-10 \mu \mathrm{m})$. Bourne et al. ${ }^{28}$ also found low agreement when comparing RNFL readings between OCT 2000 and Stratus OCT. However, the agreement also was evaluated by using the $\kappa$ coefficient to compare the RNFL classifications from both instruments. The $\kappa$ coefficient varied from 0.82 (almost perfect agreement) in the average RNFL classification with an $87.95 \%$ of cases of complete agreement to 0.15 (poor agreement) at 3 (nasal area) o'clock. In the quadrants, the highest agreement was in the inferior quadrant $(\kappa=0.71$, substantial agreement) and the lowest in the nasal area $(\kappa=0.31$, poor agreement). The $\kappa$ values and agreement obtained in the present study were 


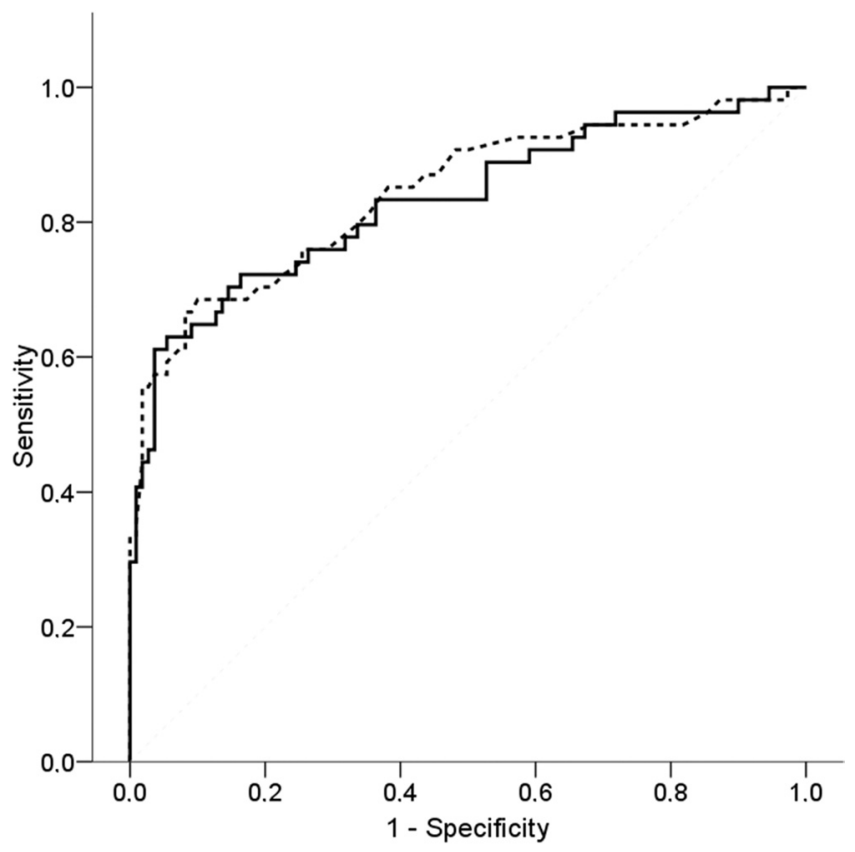

FIGURE 3. ROC curve for global RNFL thicknesses for discriminating between healthy and glaucomatous eyes with Stratus and Cirrus OCT. Solid line: Stratus OCT; dashed line: Cirrus OCT. AUCs: 0.829 (95\% CI, $0.763-0.883$ ) for Stratus OCT and 0.837 (95\% CI, $0.772-0.889$ ) for Cirrus OCT $(P=0.706)$.

similar to the agreement between the Moorfields regression analysis (MRA) comparison of the Heidelberg retinal tomograph (HRT)-3 with MRA of the HRT-2 in differentiating glaucomatous from healthy eyes. ${ }^{33}$

The present study had limitations, the first of which was the small number of cases with glaucoma after eliminating cases with a signal strength below 6 with the Stratus OCT (30\% of glaucoma cases). This small number of cases may have an influence on the AUC comparison between the groups $1 / 2$ and $3 / 4$ of the GSS classification. However, it was important to compare the sensitivity and specificity in both OCTs using only good-quality images. Second, only three scans were acquired with both OCTs, and if we obtained more scans, perhaps best-quality scans could be obtained with Stratus OCT, although patient cooperation decreases with longer examination times. Third, the order of the OCT examination was not randomized and always started with Stratus OCT. Because Stratus OCT necessitates more cooperation from the patient, we preferred to begin with the instrument with the lowest signal strength to obtain the best scan with Stratus OCT. The glaucomatous eyes included in the study have different stages of visual field damage, so the sensitivity and specificity is different in early stages of glaucoma than in more severe glaucoma; however, we compared the AUCs in two groups (initial glaucoma and advanced glaucoma), and no differences between the two OCTs were obtained. Finally, this study included only patients with confirmed visual field loss versus normal subjects. Thus, the diagnostic accuracy estimates cannot be completely applied to clinical practice because the diagnostic tests are to be used in patients with suspected disease, not patients with confirmed diagnosis. The diagnostic accuracy of both OCTs in detecting glaucoma may be overestimated. Medeiros et al. ${ }^{34}$ suggested that the accuracy of diagnostic tests in glaucoma may be largely different depending on the population studied and the reference standard used to define disease. However, the goal of the present study was to compare Stratus and Cirrus OCT in the same patients and under similar conditions. Despite these limitations, our results may contribute to the knowledge about the advantages of Cirrus OCT over Stratus OCT in glaucoma detection.

In conclusion, a comparison of the Cirrus and Stratus OCTs showed a better signal strength with the Cirrus OCT, which included more patients with a signal strength of 6 or greater. The sensitivity, specificity, and AUCs when comparing glaucomatous and normal cases were similar between the OCTs in all glaucomatous eyes, in eyes with initial glaucoma, and in eyes with more advanced glaucoma. The LRs were similar with both OCTs in global RNFL classification and varied in quadrants and clock hour sectors. The quantitative and qualitative agreements between both OCTs were better in the quadrants than in the clock hour positions, with the best agreement in global average RNFL thickness. Further studies are needed to compare both OCTs in detecting glaucoma progression.

TABLE 10. Agreement between RNFL Thickness Classification by Stratus and Cirrus OCT

\begin{tabular}{|c|c|c|c|c|}
\hline & $\begin{array}{c}\text { Complete Agreement } \\
n(\%)\end{array}$ & $\begin{array}{c}\text { Partial Agreement } \\
n(\%)\end{array}$ & $\begin{array}{c}\text { No Agreement } \\
n(\%)\end{array}$ & $\kappa$ Coefficient $(95 \%$ CI) \\
\hline Global average & $146(87.95)$ & $16(9.63)$ & $4(2.4)$ & $0.82(0.74-0.90)$ \\
\hline \multicolumn{5}{|l|}{ Quadrant } \\
\hline Superior & $135(81.32)$ & $21(12.65)$ & $10(6.02)$ & $0.66(0.54-0.77)$ \\
\hline Inferior & $135(81.32)$ & $21(12.65)$ & $10(6.02)$ & $0.71(0.62-0.81)$ \\
\hline Temporal & $130(78.31)$ & $27(16.26)$ & $9(5.42)$ & $0.49(0.36-0.63)$ \\
\hline Nasal & $148(89.15)$ & $15(9.04)$ & $3(1.81)$ & $0.31(0.10-0.52)$ \\
\hline \multicolumn{5}{|l|}{ Clock hour } \\
\hline 1 & $132(79.52)$ & $27(16.26)$ & $7(4.22)$ & $0.51(0.37-0.65)$ \\
\hline 2 & $147(88.55)$ & $16(9.63)$ & $3(1.81)$ & $0.27(0.02-0.51)$ \\
\hline 3 & $151(90.96)$ & $13(7.83)$ & $2(1.20)$ & $0.15(0-0.38)$ \\
\hline 4 & 141 (84.94) & $19(11.44)$ & $6(3.61)$ & $0.24(0.05-0.42)$ \\
\hline 5 & $133(80.12)$ & $23(13.85)$ & $10(6.02)$ & $0.52(0.38-0.65)$ \\
\hline 6 & $132(79.52)$ & $28(16.87)$ & $6(3.61)$ & $0.68(0.57-0.78)$ \\
\hline 7 & $135(81.32)$ & $23(13.85)$ & $8(4.82)$ & $0.71(0.61-0.80)$ \\
\hline 8 & $135(81.32)$ & $21(12.65)$ & $10(6.02)$ & $0.52(0.37-0.67)$ \\
\hline 9 & $139(83.73)$ & $21(12.65)$ & $6(3.61)$ & $0.46(0.30-0.62)$ \\
\hline 10 & $130(78.31)$ & $29(17.47)$ & $7(4.22)$ & $0.52(0.38-0.66)$ \\
\hline 11 & $133(80.12)$ & $26(15.66)$ & $7(4.22)$ & $0.67(0.56-0.77)$ \\
\hline 12 & $143(86.14)$ & $17(10.24)$ & $6(3.61)$ & $0.48(0.29-0.67)$ \\
\hline
\end{tabular}




\section{Acknowledgments}

The authors thank Marta García-Granero, $\mathrm{PhD}$, for valuable suggestions and assistance with the statistical analysis of the data.

\section{References}

1. Huang D, Swanson EA, Lin CP, et al. Optical coherence tomography. Science. 1991;254:1178-1181.

2. Stratus OCT Model 3000 User Manual. Dublin, CA: Carl Zeiss Meditec; 2003.

3. Lin SC, Singh K, Jampel HD, et al. American Academy of Ophthalmology; Ophthalmic Technology Assessment Committee Glaucoma Panel. Optic nerve head and retinal nerve fiber layer analysis: a report by the American Academy of Ophthalmology. Ophthalmology. 2007;114:1937-1949.

4. Cirrus HD-OCT. Details Define your Decisions. Dublin, CA: Carl Zeiss Meditec. CIR. 1595 DS-Nr. 0-1487-872.

5. Mills RP, Budenz DL, Lee PP, et al. Categorizing the stage of glaucoma from pre-diagnosis to end-stage disease. Am J Ophthalmol. 2006;141:24-30.

6. Hanley JA, McNeil BJ. A method of comparing the areas under receiver operating characteristic curves derived from the same cases. Radiology. 1983;148:839-843.

7. Bland JM, Altman DG. Statistical methods for assessing agreement between two methods of clinical measurement. Lancet. 1986;1: 307-310.

8. Cohen J. A coefficient of agreement for nominal scales. Educ Psychol Meas. 1960;20:37-46.

9. Landis JR, Koch GG. The measurement of observer agreement for categorical data. Biometrics. 1977;33:159-174.

10. Chang R, Budenz DL. New developments in optical coherence tomography for glaucoma. Curr Opin Ophthalmol. 2008;19:127135.

11. Quigley HA, Dunkelberger GR, Green WR. Retinal ganglion cell atrophy correlated with automated perimetry in human eyes with glaucoma. Am J Ophthalmol. 1989;107:453-464.

12. Sommer A, Katz J, Quigley HA, et al. Clinically detectable nerve fiber layer atrophy precedes the onset of glaucomatous field loss. Arch Ophthalmol. 1991;199:77-81.

13. Kerrigan-Baumrind LA, Quigley HA, Pease ME, et al. Number of ganglion cells in glaucoma eyes compared with threshold visual field tests in the same persons. Invest. Ophthalmol Vis Sci. 2000; 41:741-748.

14. Stratus OCT User Manual. Dublin, CA: Carl Zeiss Meditec. 2002: PN 55648 REV. A 4/03, 1-1-1-3.

15. DeLeón-Ortega JE, Arthur SN, McGwin G, et al. Discrimination between glaucomatous and nonglaucomatous eyes using quantitative imaging devices and subjective optic nerve head assessment. Invest Ophthalmol Vis Sci. 2006;47:3374-3380.

16. Hood DC, Harizman N, Kanadani FN, et al. Retinal nerve fibre thickness measured with optical coherence tomography accurately detects confirmed glaucomatous damage. Br J Ophthalmol. 2007;91:905-907.

17. Budenz DL, Michael A, Chang RT, et al. Sensitivity and specificity of the StratusOCT for perimetric glaucoma. Ophthalmology. 2005; 112:3-9.

18. Anton A, Moreno-Montañes J, Blázquez $\mathrm{F}$, et al. Usefulness of optical coherence tomography parameters of the optic disc and the retinal nerve fiber layer to differentiate glaucomatous, ocular hypertensive, and normal eyes. J Glaucoma. 2007;16:1-8.

19. Forooghian F, Cukras C, Meyerle CB, et al. Evaluation of time domain and spectral domain optical coherence tomography in the measurement of diabetic macular edema. Invest Ophthalmol Vis Sci. 2008;49:4290- 4296.

20. Gupta V, Gupta P, Singh R, et al. Spectral-domain Cirrus highdefinition optical coherence tomography is better than time-domain Stratus optical coherence tomography for evaluation of macular pathologic features in uveitis. Am J Ophthalmol. 2008;145: $1018-1022$.

21. Ahlers C, Simader C, Geitzenauer W, et al. Automatic segmentation in three-dimensional analysis of fibrovascular pigment epithelial detachment using high-definition optical coherence tomography. Br J Ophthalmol. 2008;92:197-203.

22. Wu Z, Vazeen M, Varma R, et al. Factors associated with variability in retinal nerve fiber layer thickness measurements obtained by optical coherence tomography. Ophthalmology. 2007;114:15051512.

23. Smith M, Frost A, Graham CM, et al. Effect of pupillary dilatation on glaucoma tomography assessments using optical coherence. $\mathrm{BrJ}$ Ophthalmol. 2007;91:1686-1690.

24. Savini G, Zanini M, Barboni P. Influence of pupil size and cataract on retinal nerve fiber layer thickness measurements by Stratus OCT. J Glaucoma. 2006;15:336-340.

25. Stein DM, Ishikawa H, Hariprasad R, et al. A new quality assessment parameter for optical coherence tomography. Br J Ophthalmol. 2006;90:186-190.

26. Sehi M, Guaqueta DC, Feuer WJ, et al. A comparison of structural measurements using 2 Stratus optical coherence tomography instruments. J Glaucoma. 2007;16:287-292.

27. Leal BC, Moura FC, Monteiro ML. Comparação entre o polarímetro de varredura a laser, a tomografia de coerência óptica 1 e o Stratus-OCT na detecção da perda axonal da atrofia em banda do nervo óptico. Arq Bras Oftalmol. 2006;69:531-537.

28. Bourne RR, Medeiros FA, Bowd C, et al. Comparability of retinal nerve fiber layer thickness measurements of optical coherence tomography instruments. Invest Ophthalmol Vis Sci. 2005;46: $1280-1285$.

29. Kanamori A, Nakamura M, Escano MF, et al. Evaluation of the glaucomatous damage on retinal nerve fiber layer thickness measured by optical coherence tomography. Am J Ophthalmol. 2003; 135:513-520.

30. Leung $\mathrm{CK}$, Yung WH, Ng AC, et al. Evaluation of scanning resolution on retinal nerve fiber layer measurement using optical coherence tomography in normal and glaucomatous eyes. J Glaucoma. 2004; 13:479- 485 .

31. Grimes DA, Schulz KF. Refining clinical diagnosis with likelihood ratios. Lancet. 2005;365:1500-1505.

32. Parikh RS, Parikh S, Sekhar GC, et al. Diagnostic capability of optical coherence tomography (Stratus OCT 3 ) in early glaucoma. Ophthalmology. 2007;114:2238-2243.

33. De León-Ortega JE, Sakata LM, Monheit BE, et al. Comparison of diagnostic accuracy of Heidelberg Retina Tomograph II and Heidelberg Retina Tomograph 3 to discriminate glaucomatous and nonglaucomatous eyes. Am J Ophthalmol. 2007;144:525-532.

34. Medeiros FA, Ng D, Zangwill LM, et al. The effects of study design and spectrum bias on the evaluation of diagnostic accuracy of confocal scanning laser ophthalmoscopy in glaucoma. Invest $O p h$ thalmol Vis Sci. 2007;48:214-222. 